\title{
Central Neurocytoma Presenting as Symptomatic Cataplexy
}

\author{
Jin San Lee, Gyihyaon Yun, Kyoung Jin Hwang \\ Department of Neurology, Kyung Hee University Hospital, Kyung Hee University School of Medicine, Seoul, Korea
}

Received May 13, 2020

Revised June 16, 2020

Accepted June 17, 2020

Address for correspondence Kyoung Jin Hwang, MD, $\mathrm{PhD}$ Department of Neurology, Kyung Hee University Hospital,

23 Kyungheedae-ro,

Dongdaemun-gu,

Seoul 02447, Korea

Tel: +82-2-958-8499

Fax: +82-2-958-8490

E-mail: jinie111@hanmail.net
Central neurocytomas are well-differentiated tumors and are typically found in intraventriculat lesion in adult. We report a rare case of central neurocytoma presenting as symptomatic cataplexy. A 50-year-old man visited our hospital due to repetitive drop down triggered by emotional changes and was diagnosed of central neurocytoma at right lateral ventricles and head of caudate nucleus. Alterations of striatal dopaminergic system in narcolepsy with cataplexy have been reported, but there are no reports associated with single lesion. This case suggests the striatal lesion plays a role in cataplexy. J Sleep Med 2020;17(1):98-100

Key Words: Neurocytoma, Cataplexy, Narcolepsy.

\section{Introduction}

Central neurocytoma is rare, neuronal differentiated tumors and comprise about less $1 \%$ of intracranial tumors. Central neurocytoma is usually located within the ventricular system and presents symptom as increasing obstructive hydrocephalus. The most common symptom is headache, dizziness, nausea, vomiting, papilledema, and ataxia by increased intracranial pressure. ${ }^{1}$ Here we report a rare case of central neurocytoma presenting as symptomatic cataplexy.

\section{Case Report}

A 50-year-old man with a history of hypertension and hyperlipidemia visited our clinic complaining of repetitive drop down, which started six months ago. The paralytic attacks were mainly triggered by emotional changes. He has been managing a billiard room for 10 years and had very good billiard skills. When the patient concentrates on a very difficult 3-balls billiards game and solves the problem by hitting the ball correctly, a paralytic attack has occurred followed by a virtuous feeling. He also complained of mild excessive daytime sleepiness from three years ago. Epworth sleepiness scale was 8 and Stanford sleepiness scale 3. There was no preceding history of en-

This is an Open Access article distributed under the terms of the Creative Commons Attribution Non-Commercial License (https://creativecommons.org/licenses/by-nc/4.0) which permits unrestricted non-commercial use, distribution, and reproduction in any medium, provided the original work is properly cited. cephalitis, antipsychotic medication use, or exposure to toxic substances. A neurological examination was normal.

Since the patient's symptoms occurred in abruptly repeated pattern triggered by emotional changes during wakefulness, cataplexy was suspected, and a laboratory and imaging diagnostic workup was performed. There were no significant laboratory abnormalities including serology and hematological tests, thyroid function tests, rapid plasma reagin, and Treponema pallidum hemagglutination assays. An electroencephalogram was reported as normal. However, brain magnetic resonance imaging demonstrated tumor with calcification occupying right lateral ventricles on fluid-attenuated inversion recovery, T1-weighted imaging, and susceptibility-weighted imaging sequences (Fig. 1A-C). In addition, there was a calcified lesion at the right head of caudate nucleus on brain computed tomography of the patient (Fig. 1D, E). We tried to perform a polysomnography for further evaluation, he refused the examination and we lost to additional follow-up.

\section{Discussion}

To the best of our knowledge, a central neurocytoma presenting as symptomatic cataplexy has never been reported. Central neurocytomas are well-differentiated tumors and are typically found in the lateral or third ventricle, attached to the septum pellucidum or ventricular wall at the foramen of Monro. ${ }^{2}$ Most patients present with symptoms of increased intra- 

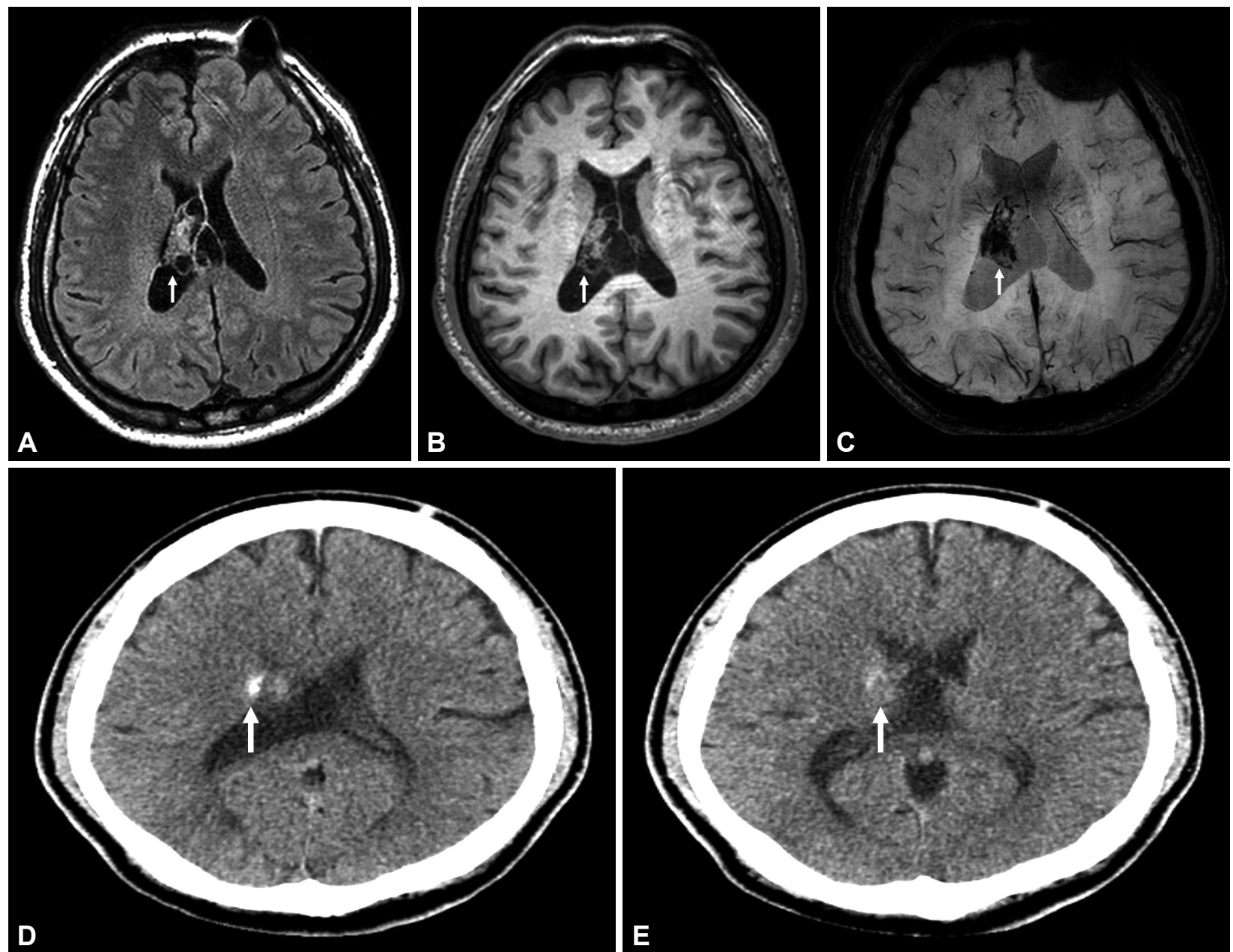

Figure 1. Tumor with calcification occupying right lateral ventricles on fluid-attenuated inversion recovery (A), T1-weighted imaging (B), and susceptibility-weighted imaging sequences $(C)$ of brain MRI. Calcified lesion at the right head of caudate nucleus on brain computed tomography of the patient $(D, E)$.

cranial pressure due to hydrocephalus such as headache, dizziness, and visual disturbances, but focal neurologic deficits are uncommon.

Cataplexy is characterized as sudden involuntary muscle weakness or paralysis during wakefulness, typically triggered by strong emotions, and is the indispensable symptom of narcolepsy with cataplexy. ${ }^{3}$ It has been known that cataplexy occurs almost exclusively in patients with depletion of hypothalamic hypocretin neurons. Although the fascinating link between emotion and cataplexy remains unclear, the hypocretin system has strong reciprocal links with limbic areas such as the dopaminergic ventral tegmental area and the amygdala. These areas primarily involved in reward and emotional processing. ${ }^{4}$ Patient with cataplexy has been known to have abnormal activity in reward brain circuit. ${ }^{5}$

Alterations of striatal dopaminergic system in narcolepsy with cataplexy also have been reported. In functional brain study, patients with narcolepsy showed increased striatal D2 receptor binding and a positive correlation between this and the frequency of cataplexy and sleep attack. ${ }^{6}$ Besides, in cerebral perfusion measured by brain single photon emission computed tomography showed hypoperfusion in bilateral caudate nuclei. ${ }^{7}$ In patient with cataplexy, the hypothalamus is the most common region, but there are no reports associated with single brain lesion, except multiple white matter lesion in patient with multiple sclerosis. ${ }^{8}$ The limitation of this case is lack of histological confirmation and clinical change due to no surgery. Through this case, we suggest the striatal lesion might play a role in cataplexy.

\section{Acknowledgments}

None.

\section{Conflicts of Interest}

The authors have no potential conflicts of interest to disclose.

\section{ORCID iDs}

Jin San Lee

Guihyaon Yun

https://orcid.org/0000-0002-5017-854X

https://orcid.org/0000-0002-2436-4947 
Kyoung Jin Hwang https://orcid.org/0000-0003-0856-2203

\section{Author Contributions}

Conceptualization: Jin San Lee. Data curation: Jin San Lee, Kyoung Jin Hwang. Formal analysis: Guihyaon Yun. Investigation: Guihyaon Yun. Methodology: Jin San Lee. Supervision: Kyoung Jin Hwang. Writingoriginal draft: Jin San Lee, Kyoung Jin Hwang. Writing_review \& editing: Kyoung Jin Hwang.

\section{REFERENCES}

1. Evangelista E, Lopez R, Dauvilliers Y. Update on treatment for idiopathic hypersomnia. Expert Opin Investig Drugs 2018;27:187-192.

2. Sharma MC, Deb P, Sharma S, Sarkar C. Neurocytoma: a comprehensive review. Neurosurg Rev 2006;29:270-285; discussion 285.
3. Dauvilliers Y, Siegel JM, Lopez R, Torontali ZA, Peever JH. CataplexyClinical aspects, pathophysiology and management strategy. Nat Rev Neurol 2014;10:386-395.

4. Haghparast A, Fatahi Z, Arezoomandan R, Karimi S, Taslimi Z, Zarrabian S. Functional roles of orexin/hypocretin receptors in reward circuit. Prog Brain Res 2017;235:139-154.

5. Reading P. Cataplexy. Pract Neurol 2019;19:21-27.

6. Eisensehr I, Linke R, Tatsch K, et al. Alteration of the striatal dopaminergic system in human narcolepsy. Neurology 2003;60:1817-1819.

7. Joo EY, Hong SB, Tae WS, et al. Cerebral perfusion abnormality in narcolepsy with cataplexy. Neuroimage 2005;28:410-416.

8. Kallweit U, Bassetti CLA, Oberholzer M, et al. Coexisting narcolepsy (with and without cataplexy) and multiple sclerosis: six new cases and a literature review. J Neurol 2018;265:2071-2078. 